\title{
Combining Diffusion NMR and Small-Angle Neutron Scattering Enables Precise Measurements of Polymer Chain Compression in a Crowded Environment
}

\author{
Swomitra Palit, ${ }^{1}$ Lilin He, ${ }^{2}$ William A. Hamilton, ${ }^{3}$ Arun Yethiraj, ${ }^{4}$ and Anand Yethiraj ${ }^{1, *}$ \\ ${ }^{1}$ Department of Physics and Physical Oceanography, Memorial University, \\ St. John's, Newfoundland AlB3X7, Canada \\ ${ }^{2}$ Biology and Soft Matter Division, Neutron Sciences Directorate, Oak Ridge National Laboratory, \\ Oak Ridge, Tennessee 37831, USA \\ ${ }^{3}$ Instrument and Source Division, Neutron Sciences Directorate, Oak Ridge National Laboratory, \\ Oak Ridge, Tennessee 37831, USA \\ ${ }^{4}$ Department of Chemistry, University of Wisconsin, Madison, Wisconsin 53706, USA
}

(Received 20 September 2016; revised manuscript received 2 February 2017; published 3 March 2017)

The effect of particles on the behavior of polymers in solution is important in a number of important phenomena such as the effect of "crowding" proteins in cells, colloid-polymer mixtures, and nanoparticle "fillers" in polymer solutions and melts. In this Letter, we study the effect of spherical inert nanoparticles (which we refer to as "crowders") on the diffusion coefficient and radius of gyration of polymers in solution using pulsed-field-gradient NMR and small-angle neutron scattering (SANS), respectively. The diffusion coefficients exhibit a plateau below a characteristic polymer concentration, which we identify as the overlap threshold concentration $c^{\star}$. Above $c^{\star}$, in a crossover region between the dilute and semidilute regimes, the (long-time) self-diffusion coefficients are found, universally, to decrease exponentially with polymer concentration at all crowder packing fractions, consistent with a structural basis for the long-time dynamics. The radius of gyration obtained from SANS in the crossover regime changes linearly with an increase in polymer concentration, and must be extrapolated to $c^{\star}$ in order to obtain the radius of gyration of an individual polymer chain. When the polymer radius of gyration and crowder size are comparable, the polymer size is very weakly affected by the presence of crowders, consistent with recent computer simulations. There is significant chain compression, however, when the crowder size is much smaller than the polymer radius gyration.

DOI: 10.1103/PhysRevLett.118.097801

Introduction.-The cell is a dense mixture of a number of different components including flexible (e.g., nucleic acids) and globular (e.g., proteins) macromolecules. There has been considerable recent interest on these "crowding" effects, i.e., the effect of inert macromolecules on the conformational properties of flexible molecules, and on the reaction between enzymes and substrates [1,2]. Similar physics is expected in seemingly different problems such as colloid-polymer mixtures, where the addition of polymers can either stabilize or destabilize a colloidal dispersion [3-5], or nanoparticle polymer composites [6], where the properties of the composite are sensitive to the nature of particle-polymer interactions. In this Letter we are concerned with the effect of inert spherical particles-which, following the biophysics community, we refer to as "crowders"- on the conformational properties and dynamics of polymers in solution.

Entropy, via the excluded volume effect, is an important ingredient in macromolecular crowding [1,2], although nonspecific chemical interactions can also play a role $[7,8]$. From a colloid- and polymer-science perspective, a quantitative understanding of the entropic contributions to crowding is a necessary precondition to an understanding of the broader crowding problem. Even in the absence of other interactions, the osmotic pressure of the crowder (radius $R_{c}$ ) can alter the size of a flexible macromolecule (radius of gyration $R_{g}$ ). Entropy can give rise to attractions due to depletion forces in both the "colloid limit" $\left(\lambda=R_{g} / R_{c} \ll 1\right)$ [9] and the "protein limit" $(\lambda \gg 1)$ [10]. In a simple model system, i.e., a colloid-polymer mixture consisting of a flexible polymer (i.e., macromolecule) and compact crowder $[11,12]$, one thus qualitatively expects a compression of polymer chains for large $\lambda$. On the other hand, a recent study suggests [13] that changing crowder size has a weak effect. It is safe to say that we still do not have a quantitative understanding of the differences between various simulations and experiments on the degree of compression.

We emphasize the distinction between the focus of this Letter, which is on the effect of spherical particles on the properties of the polymers, and the focus of work in colloid-polymer mixtures [3-5] and polymer nanocomposites [6], which is on the effect of polymers on the interaction between the particles. While polymers can induce either a net attraction or repulsion between particles (depending on the interactions and polymer concentration), the effect of particles on polymers is generally compressive when the particles are mobile, 


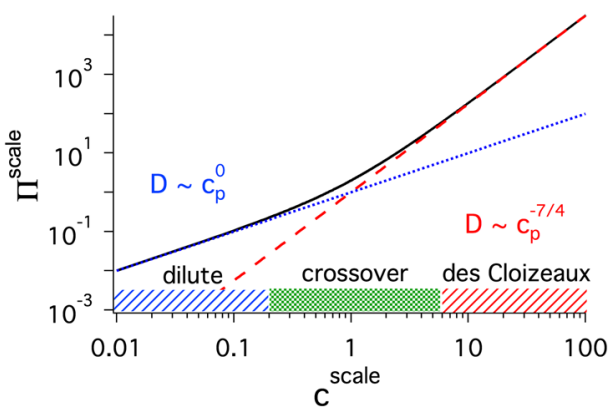

FIG. 1. The osmotic pressure of pure PEG in water (no crowder) is well described by a phenomenological scaling form (solid black line, from Cohen et al. [25]). The solution is dilute when $c^{\text {scale }} \equiv c_{p} / c^{\#} \leq 0.2$, and in the semidilute entangled regime when $c^{\text {scale }} \geq 6$ (dashed red line): the crossover regime (green hashed region) is in between $\left(0.2<c^{\text {scale }}<6\right)$. The expected scaling of the self-diffusion coefficient in the dilute (blue hashed) and semidilute (red hashed) regimes [30] is $D \sim c_{p}^{0}$ and $D \sim c_{p}^{-7 / 4}$.

although the situation is more complicated if the positions of the particles is quenched $[14,15]$.

Computer simulations of crowding employing a variety of simulation methodologies [11,12,16-20] indicate that crowding decreases the radius of gyration for the unfolded state, but quantitatively the results are mixed even for simple systems: for example, a polymer-nanoparticle simulation with $\lambda \sim 1$ found that the polymer chains collapsed into a compact globular form with an overall decrease of $R_{g}$ by $20 \%$ for $\Phi_{c}=0.35$ [11], while another simulation found more modest chain compression of about $5 \%$ [12].

Intrinsically disordered proteins (IDPs) are flexible biopolymers that should be influenced by crowding. Two small-angle neutron scattering (SANS) experiments found that IDPs exhibited a varying degree of compression for similar $\lambda$ : in one study, a $13 \%$ size decrease resulted at a moderate crowder packing fraction $\Phi_{c}=0.07$ [21], while in another only a $6 \%$ reduction of $R_{g}$ was seen for $\Phi_{c} \sim 0.20$ [22]. Single-molecule Förster resonance energy transfer spectroscopy was used to quantify IDP size, using a linear polymer, polyethylene glycol (PEG), as a crowder. Here, IDP chain compression was observed to be stronger with larger crowders (i.e., with $\lambda$ closer to 1) [23]; in other words, IDPs in a polymer solution, i.e., in an extended polymeric crowder, do the opposite of a compact crowder.

In order to make quantitative the comparisons between simulation and experiment, a useful model system employs PEG as polymeric macromolecule and a polysucrose (Ficoll70) as nanoparticle spherical crowder [24]. For $\lambda \sim 1$ and $\Phi_{c}=0.25$, the PEG was reported to undergo significant chain compression to $50 \%$ of its size in free solution, significantly more than simulations have found [11,12]. In these SANS experiments [24], isolated chain behavior was extracted from experiments at finite concentrations $\left(0.004 \mathrm{~g} / \mathrm{cm}^{3}<c_{p}<0.03 \mathrm{~g} / \mathrm{cm}^{3}\right)$ by linear extrapolation to $c_{p}=0$. Figure 1 demonstrates why such an experimental extrapolation is problematic. The osmotic pressure of a neutral flexible polymer in solution may be written down as a phenomenological sum of scalings as a function of a scaled polymer concentration $c^{\#}$ [25]. The scaled osmotic pressure (details in the Supplemental Material [26]) obeys a universal behavior as a function of the scaled concentration. In terms of scaled concentrations, the SANS experiments [24] were carried out in the range $0.2<c^{\text {scale }}<2$ in Fig. 1 , and are thus completely in the (green hashed) crossover regime. It is thus wise to carry out experimental measurements to low-enough concentrations in order to determine the overlap concentration $c^{\star}$ below which the polymer is dilute. In the current work, experiments are carried out in the range $0.015<c^{\text {scale }}<2$.

The strength of the current Letter is that we combine SANS measurements of polymer size with self-diffusion measurements via pulsed-field-gradient (PFG) NMR. The latter can conveniently be carried out to much lower polymer concentrations $c_{p}$ than the SANS measurements, enabling clear estimates of $c^{\star}$ at different crowder packing fractions. We then use this to obtain reliable estimates of $R_{g}\left(0, \Phi_{F}\right)$ that we compare with simulations.

Polymer self-diffusion.-PFG NMR diffusion measurements were carried out for aqueous PEG-Ficoll70 solutions in water prepared for PEG concentrations between 0.0003 and $0.03 \mathrm{~g} / \mathrm{cm}^{3}$ and Ficoll 70 volume fractions $\Phi_{F}$ between 0 and 0.30 . The details of the PFG NMR method were reviewed recently [31] and are summarized in the Supplemental Material [26]. The dashed blue line in Fig. 2(a) shows the diffusion coefficient of the last sample in a solution series that did not phase separate.

The PEG diffusion coefficient $D\left(c_{p}, \Phi_{F}\right)$, shown in Fig. 2(a) with a logarithmic scale on the ordinate, is constant for low $c_{p}$. For all $\Phi_{F}$, the self-diffusion coefficient decreases sharply above a ( $\Phi_{F}$-dependent) characteristic concentration which we identify with $c^{\star}$. A piecewise fit of $D\left(c_{p}, \Phi_{F}\right)$ as a function of $c_{p}$,

$D\left(c_{p}, \Phi_{F}\right)=D\left(0, \Phi_{F}\right), \quad c_{p} \leq c^{\star}$,
$D\left(c_{p}, \Phi_{F}\right)=D^{\star}\left(\Phi_{F}\right) \exp \left(-c_{p} / c_{2}\right), \quad c_{p}>c^{\star}$,

with exponential dependence above $c^{\star}$ and a plateau value below, provides a good fit to all the results. From this, we extract the dilute-limit diffusion coefficient $D\left(0, \Phi_{F}\right)$ as well as $c^{\star}$ and a second characteristic concentration $c_{2}$ for each Ficoll70 volume fraction $\Phi_{F} . D^{\star}\left(\Phi_{F}\right)=$ $D\left(0, \Phi_{F}\right) \exp \left(c^{\star} / c_{2}\right)$ is not fit, but evaluated from continuity at $c^{\star}$.

For $\Phi_{F}=0$, referring to Fig. 1, we expect $D\left(c_{p}, \Phi_{F}=\right.$ $0) \sim c_{p}^{0}$ in the dilute (blue hashed) regime and $D \sim c_{p}^{-7 / 4}$ in the des Cloizeaux (also referred to as the semidilute entangled) regime [30]. As a higher-order correction, the friction coefficient is expected to show a linear dependence 

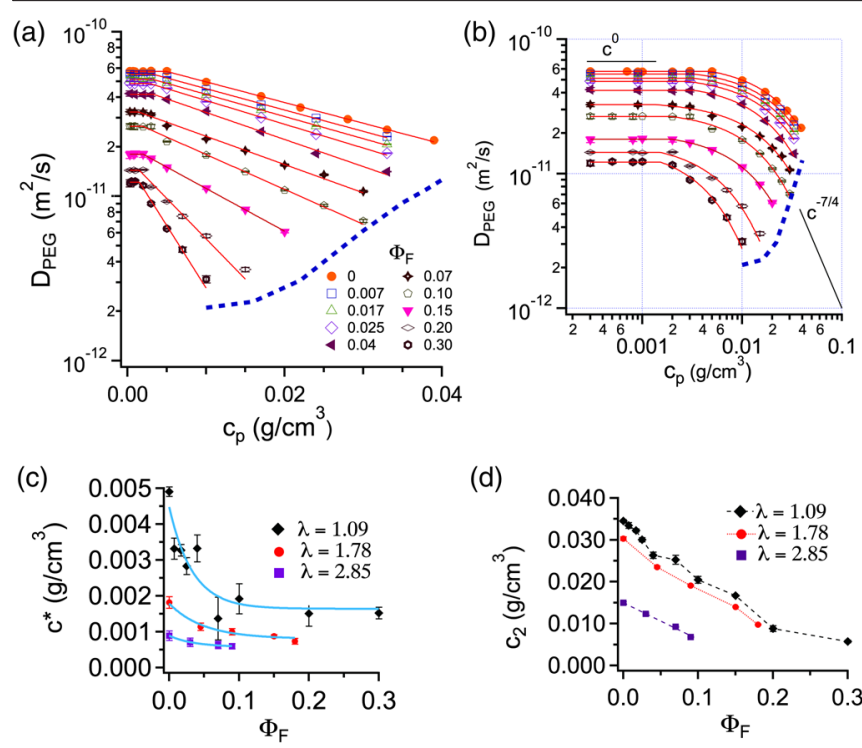

(d)

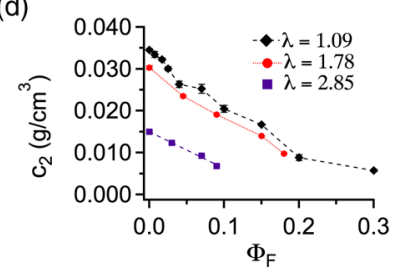

FIG. 2. Self-diffusion: (a) Diffusion coefficient of PEG $\left(M_{w}=20000\right)$ polymer in water as a function of polymer concentration $c_{p}$, in the absence of the crowder, Ficoll70, as well as for several Ficoll70 volume fractions $\Phi_{F}\left(\lambda=R_{g} /\right.$ $R_{c}=1.09$ ). A good fit to exponential behavior is possible, in all cases, above a characteristic PEG concentration, $c^{\star}$, with an extrapolated value $D^{\star}$. Below this PEG concentration, a plateau is observed at $D\left(0, \Phi_{F}\right)$. (b) A log-log plot of $D_{\mathrm{PEG}}$ vs $c_{p}$ shows the plateau, and also that a power law cannot fit the data in the crossover regime. (c) Dependence of characteristic PEG concentration $c^{\star}$ as a function of $\Phi_{F}$ for three polymer molecular weights $\left(M_{w}=20000, M_{w}=42800\right.$, and $\left.M_{w}=132000\right)$, corresponding to $\lambda=1.09,1.78$, and 2.85 . The solid blue curves may be treated as a guide to the eye. (d) From the phenomenological exponential decay in (a), a second characteristic concentration $c_{2}$ is obtained (for each $\Phi_{F}$ ).

on polymer concentration due to the occasional interactions of polymer chains in the dilute regime, but this dependence has been seen to be very weak for short-chain polymers [32]. The log-log representation in Fig. 2(b) shows, first, that the plateau regime is well defined in all cases, and second, that a power law cannot fit. While the presence of a plateau for all $\Phi_{F}$ is experimentally clear, the reason for it is not obvious: it signifies that a dilute polymer limit continues to exist in the limit of crowding.

Figure 2(c) shows that $c^{\star}$ is a decreasing function of $\Phi_{F}$, not only for $M_{w}=20000\left(\lambda=R_{g} / R_{c}=1.09\right)$, but also for two other longer polymers $\left(M_{w}=42800\right.$ and 132000 , see Supplemental Material [26] Fig. S2), corresponding to $\lambda=1.78$ and 2.85 . For all polymer molecular weights and crowder volume fractions, there is a characteristic polymer concentration $c^{\star}$ below which the dynamics is independent of polymer concentration: the "polymer-dilute" regime. In addition, in all cases, $c^{\star}$ changes little beyond $\Phi_{F} \sim 0.1$, perhaps indicative of the onset of crowding.

From the exponential decay in Fig. 2, we also obtain a second characteristic concentration $c_{2}$; e.g., for $\lambda=1.09$,

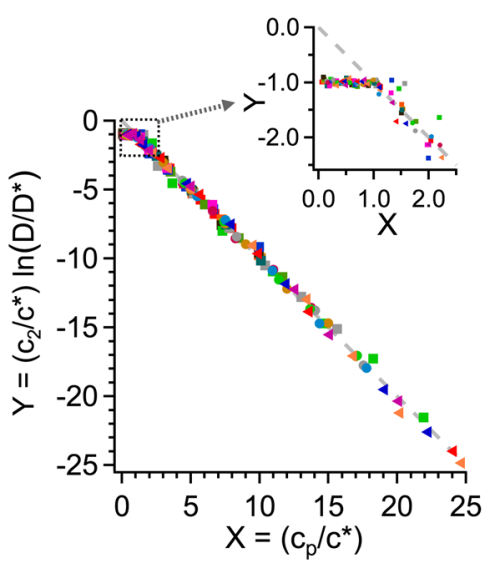

FIG. 3. Universal behavior in the dynamics: Using the values $D^{\star}, c^{\star}$, and $c_{2}$ (all functions of only $\Phi_{F}$ ) from each fit, all the diffusion results (as a function of $c_{p}$ and $\Phi_{F}$ ) are replotted in dimensionless form, $Y=\left(c_{2} / c^{\star}\right) \ln \left[D\left(c_{p}, \Phi_{F}\right) / D^{\star}\right]$ as a function of a scaled polymer concentration $X=c_{p} / c^{\star}$. For all three polymers, there is good collapse onto one master plot that shows a sharp transition at $X=1$ (see inset) from a polymer-dilute plateau to an exponential concentration dependence of the diffusion coefficient.

$c_{2}$ decreases from 0.035 to $0.005 \mathrm{~g} / \mathrm{cm}^{3}$ as $\Phi_{F}$ approaches the crowding limit [Fig. 2(d)], which implies a sevenfold increase in "effective" PEG concentration for $\Phi_{F}=0.3$. Physically, the value of $c_{2}$ appears consistent with the end of the crossover regime. The decrease in $c_{2}$ with $\Phi_{F}$ is consistent, too, with the decrease in the observed phase separation concentration as $\Phi_{F}$ increases [dashed blue line in Fig. 2(a)].

Using the fitted $c^{\star}$ and $c_{2}$, as well as $D^{\star}\left(\Phi_{F}\right)$, we plot a scaled and dimensionless version of the self-diffusion coefficients $Y=\left(c_{2} / c^{\star}\right) \ln \left(D / D^{\star}\right)$ as a function of a scaled polymer concentration $X=c_{p} / c^{\star}$. From the model equation (1), $Y=-1$ for $X \leq 1$ and $Y=-X$ for $X>1$. Figure 3 shows a master plot of all data for all three polymers: clearly, both the plateau below $c^{\star}$ and the exponential dependence above $c^{\star}$ are universally valid in the crossover regime at all $\lambda$ and $\Phi_{F}$.

Why is there no power-law scaling, even for pure polymer? Indeed, there is clear experimental indication (Callaghan and Pinder [32]) that power-law scaling fails for short-chain polymers. It is also possible [see Fig. 2(b) and Supplemental Material [26], Fig. S2] that there could be power-law scaling at large concentrations (the red-hashed regime in Fig. 1) if this was accessible in the presence of crowding. Why the exponential dependence? Rosenfeld [33] and Dzugutov [34] have proposed an exponential relationship between atomic diffusion and the excess entropy $S_{2} / k_{B}$ (in the two-particle approximation); moreover, recent 2D simulations and colloid experiments [35] show that $S_{2} / k_{B}$ is proportional to the colloid packing fraction for packing fractions less than 0.4. Because of the PEG's conformational degrees of freedom, it is inadvisable 


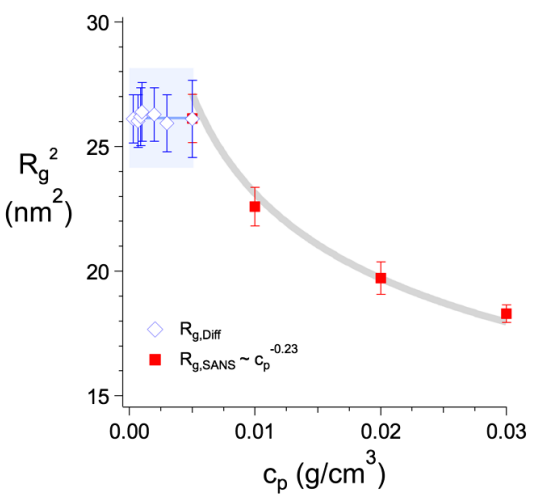

FIG. 4. Diffusion vs SANS: A comparison of $R_{g}^{2}$ from diffusion (blue, $\left.c_{p}<0.005 \mathrm{~g} / \mathrm{cm}^{3}\right)$ and SANS $\left(c_{p}>0.005 \mathrm{~g} / \mathrm{cm}^{3}\right)$ for PEG-water solution $\left(\Phi_{F}=0\right)$ confirms that the overlap concentration deduced from diffusion $\left(c_{\text {Diff }}^{\star}=0.005 \mathrm{~g} / \mathrm{cm}^{3}\right)$ is also meaningful as the thermodynamic overlap concentration $c^{\star}$.

to invoke the two-particle excess entropy; nevertheless, it is reasonable to believe that the exponential dependence of the long-time self-diffusion coefficient at all $\Phi_{F}$ is purely structural in origin. To our knowledge, this is the first evidence of this in three dimensions.

Polymer size.-Next, we describe our SANS measurements for d-PEG-Ficoll70 solutions (carried out at the High Flux Isotope Reactor at Oak Ridge National Laboratories on the GP-SANS instrument [36]) for PEG concentrations between 0.001 and $0.03 \mathrm{~g} / \mathrm{cm}^{3}$ and Ficoll70 volume fractions between 0 and 0.3 . In contrast with $\mathrm{PFG}$ NMR, measurements at lower polymer concentrations were impractical due to long acquisition times. Polymer radius of gyration $R_{g}$ was obtained in two ways, by a fit of the $q$-dependent scattering intensity to the Debye model as well as by fitting the low- $q$ scattering intensity using the Guinier approximation. Both gave consistent $R_{g}$; an example is shown in the Supplemental Material [26] (Fig. S1). Consistent with the previously reported SANS study of Le Coeur et al. [24], Fig. 5(a) shows that for pure PEG and water, there is a decrease in $R_{g}$ with increasing PEG concentration, while at $\Phi_{F}=0.1,0.15,0.2$ and 0.3 , there is an increase. When each data set is fit to a straight line, a linear extrapolation at each Ficoll70 concentration suggests a convergence at nonzero $c_{p}$, around $c_{p} \sim 0.003 \mathrm{~g} / \mathrm{cm}^{3}$. Quantitatively, the linear-extrapolated $R_{g}^{\star}$ in our Letter [denoted by a star symbol in Fig. 5(a)] and those of Le Coeur et al. are in rough agreement for low $\Phi_{F}$ but deviate at $\Phi_{F} \geq 0.2$ (a comparison is shown in Supplemental Material [26] Fig. S3). In both cases, linear extrapolation would imply a decrease in size of isolated polymer chains due to increasing $\Phi_{F}$. The self-diffusion measurements, however, have demonstrated clearly that such an extrapolation from the crossover regime $\left(c_{p}>c^{\star}\right)$ to the dilute (plateau) regime $\left(c_{p}<c^{\star}\right)$ is not valid. We identify the $c^{\star}$ obtained from diffusion measurements with the thermodynamic overlap concentration. The validity of this identification is shown in Fig. 4, which shows (for $\Phi_{F}=0$ ) that $R_{g}^{2}$ from diffusion at lower polymer concentrations $c_{p}$ and SANS mostly at higher $c_{p}$ converge at a common $c^{\star}$; this is further discussed in Supplemental Material [26]. Since the self-diffusion coefficient is unchanging in the polymerdilute regime, $R_{g}$ must therefore also be constant. Simulations [37] have also observed that the polymer $R_{g}(0,0)$ (no crowder) is essentially constant in the dilute limit.

In Fig. 5(a), we plot $R_{g}$ as a function of $c_{p}$ for the different $\Phi_{F}$, and employ a piecewise linear fit,

$$
\begin{aligned}
& R_{g}\left(c_{p}, \Phi_{F}\right)=R_{g}\left(0, \Phi_{F}\right), \quad c_{p} \leq c^{\star}, \\
& R_{g}\left(c_{p}, \Phi_{F}\right)=R_{g}\left(0, \Phi_{F}\right)+m\left(\Phi_{F}\right)\left(c_{p}-c^{\star}\right), \quad c_{p}>c^{\star},
\end{aligned}
$$

with slope $m\left(\Phi_{F}\right)$, and a plateau value $R_{g}\left(0, \Phi_{F}\right)$ at and below $c_{p}=c^{\star}$, i.e., in the polymer-dilute limit. $c^{\star}$ itself is not a fit parameter, since we have it from the diffusion measurements carried out to much lower $c_{p}$. In the absence
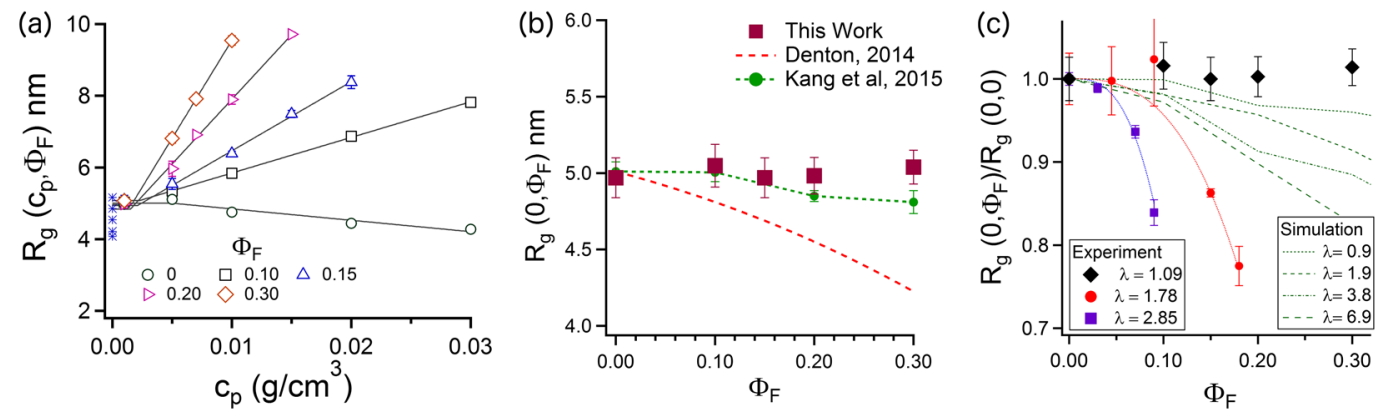

FIG. 5. SANS: (a) $R_{g}\left(c_{p}, \Phi_{F}\right)$ vs polymer concentration $c_{p}$ for $\lambda=1.09$. $R_{g}$ is fit to Eq. (2) assuming that the $c^{\star}$ is the same as for the diffusion experiment. The blue asterisks reflect the value that would be obtained by a naive extrapolation of $R_{g}$ from the crossover regime. (b) The fit shows that $R_{g}\left(0, \Phi_{F}\right)$ in the "polymer-dilute" limit exhibits at most a weak dependence on Ficoll70 volume fraction $\Phi_{F}$. (c) $R_{g}$ dependence on packing fraction $\Phi_{F}$ for three polymer molecular weights, corresponding to $\lambda=1.09,1.78$, and 2.85 respectively. Results are compared with simulations from Kang et al. [12]. 
of crowders, we can calculate the ratio $R_{g}(0,0) / R_{H}$ $\left(R_{g}(0,0)\right.$ is obtained from SANS and $R_{H}$ from $D(0,0)$ and the Stokes-Einstein relation: we obtain a value $R_{g}(0,0) / R_{H}=1.18 \pm 0.04$ consistent with renomalization group calculations [38].

The resulting $R_{g}\left(0, \Phi_{F}\right)$, for $\lambda=1.09$, is shown in Fig. 5(b). This $R_{g}$ value is remarkably insensitive to the Ficoll70 volume fraction, and is quantitatively consistent with the simulations of Kang et al. [12], but not with the simulation and free-volume theory of Denton and co-workers [11]. This quantitative agreement signals that Ficoll70 is an inert crowder in this experiment. Identifying macromolecule-crowder interactions is a key challenge in the study of macromolecular crowding, and this is therefore significant.

Interestingly, $R_{g}$ in the dilute polymer limit is insensitive to $\Phi_{F}$ [Fig. 5(b)], but the value of the overlap concentration $c^{\star}$ is not [Fig. 2(c)]. At first glance this might seem strange because $c^{\star} \sim N / R_{g}^{3}$ where $N$ is the number of monomers. Note, however, that this relation comes from equating the "internal concentration" of the polymer $\left(N / R_{g}^{3}\right)$ to the overall concentration of pure polymer solutions; at $c^{\star}$ different molecules begin to touch each other on average. The presence of crowders decreases the volume available to polymers and, therefore, as $\Phi_{F}$ increases one expects $c^{\star}$ to decrease: the internal concentration is still $N / R_{g}^{3}$, but the relevant overall concentration is not the polymer concentration. Using an effective medium or mean-field model, one would expect $c^{\star}$ to decrease linearly with $\Phi_{F}$ at low values of $\Phi_{F}$. We do not have a good structural explanation for why $c^{\star}$ is insensitive to $\Phi_{F}$ for large values of $\Phi_{F}$, but we note that at high $\Phi_{F}$ the system is closer to phase separation, and the crowder structure might be heterogeneous.

Varying the macromolecule-crowder size ratio.-Next, we use the $c^{\star}$ obtained from our diffusion studies for the two polymers with larger molecular weights $(\lambda=1.78$ and 2.85 ) in order to reanalyze preexisting SANS results [39]. The overlap concentration obtained from diffusion measurements decreases substantially with increasing $\lambda$ [Fig. 2(b)]. Figure 5(c) shows that the radius of gyration exhibits significant compression as a function of $\Phi_{F}$. While the data at $\lambda \sim 1$ are close to those from simulation at a similar $\lambda$ [Fig. 5(c)], those for higher $\lambda$ show much stronger compression than predicted by simulation.

Conclusion.-We have obtained polymer size as well as diffusion coefficients of a polymer-crowder solution using SANS and PFG NMR in tandem, as a function of the crowder packing fraction $\Phi_{F}$. The spectral selectivity of PFG NMR provides us with the sensitivity to measure diffusion coefficients at low polymer concentrations. These diffusion measurements unambiguously establish that there exists a polymer-dilute regime, $c^{\star}\left(\Phi_{F}\right)$, for each crowder packing fraction. One upshot of this study is that the polymer (polyethylene glycol), in the dilute limit, appears to be unchanged in hydrodynamic size even at relatively large crowder (Ficoll70) volume fractions for polymercrowder size ratio $\lambda \sim 1$, as predicted in the simulation of Kang et al. [12].

In contrast with $\lambda=1.09$, we find significant chain compression for isolated polymers when $\lambda$ is 1.78 and 2.85 , respectively. This is much more pronounced than the predictions from simulation; however, the 10\%-15\% reduction in $R_{g}$ at $\Phi_{F}=0.1$ for $\lambda=2.85$ is close in magnitude to experimental observations in IDPs [21].

This work was supported by the Natural Sciences and Engineering Research Council of Canada. A portion of this research used resources at the High Flux Isotope Reactor, a DOE Office of Science User Facility operated by the Oak Ridge National Laboratory. We acknowledge Mohana Yethiraj for advice and useful discussions.

*ayethiraj@mun.ca

[1] S. B. Zimmerman and A. P. Minton, Annu. Rev. Biophys. Biomol. Struct. 22, 27 (1993).

[2] A. P. Minton, J. Biol. Chem. 276, 10577 (2001).

[3] A. P. Gast, C. K. Hall, and W. B. Russel, J. Colloid Interface Sci. 96, 251 (1983).

[4] R. Dickman and A. Yethiraj, J. Chem. Phys. 100, 4683 (1994).

[5] A. Yethiraj, C. K. Hall, and R. Dickman, J. Colloid Interface Sci. 151, 102 (1992).

[6] D. Banerjee and K. S. Schweizer, J. Chem. Phys. 142, 214903 (2015).

[7] H. X. Zhou, G. Rivas, and A.P. Minton, Annu. Rev. Biophys. 37, 375 (2008).

[8] M. Sarkar, A. E. Smith, and G. J. Pielak, Proc. Natl. Acad. Sci. U.S.A. 110, 19342 (2013).

[9] S. Asakura and F. Oosawa, J. Chem. Phys. 22, 1255 (1954).

[10] K. J. Mutch, J. S. van Duijneveldt, and J. Eastoe, Soft Matter 3, 155 (2007).

[11] A. R. Denton, Int. Rev. Cell Mol. Biol. 307, 27 (2014).

[12] H. Kang, P. A. Pincus, C. Hyeon, and D. Thirumalai, Phys. Rev. Lett. 114, 068303 (2015).

[13] K. A. Sharp, Proc. Natl. Acad. Sci. U.S.A. 112, 7990 (2015).

[14] R. W. Chang and A. Yethiraj, Phys. Rev. Lett. 96, 107802 (2006).

[15] B. J. Sung, R. Chang, and A. Yethiraj, J. Chem. Phys. 130, 124908 (2009).

[16] A. P. Minton, Biophys. J. 88, 971 (2005).

[17] D. Tsao and N. V. Dokholyan, Phys. Chem. Chem. Phys. 12, 3491 (2010).

[18] N. A. Denesyuk and D. Thirumalai, J. Am. Chem. Soc. 133, 11858 (2011).

[19] S. Qin and H.-X. Zhou, J. Phys. Chem. Lett. 4, 3429 (2013).

[20] N. A. Denesyuk and D. Thirumalai, Biophys. Rev. Lett. 5, 225 (2013).

[21] D. Johansen, C. M. Jeffries, B. Hammouda, J. Trewhella, and D. P. Goldenberg, Biophys. J. 100, 1120 (2011).

[22] D. Goldenberg and B. Argyle, Biophys. J. 106, 905 (2014). 
[23] A. Soranno, I. Koenig, M. B. Borgia, H. Hofmann, F. Zosel, D. Nettels, and B. Schuler, Proc. Natl. Acad. Sci. U.S.A. 111, 4874 (2014).

[24] C. Le Coeur, B. Demé, and S. Longeville, Phys. Rev. E 79, 031910 (2009).

[25] J. A. Cohen, R. Podgornik, P. L. Hansen, and V. A. Parsegian, J. Phys. Chem. B 113, 3709 (2009).

[26] See Supplemental Material at http://link.aps.org/ supplemental/10.1103/PhysRevLett.118.097801, which includes Refs. [27-29], for more details of the materials and methods, the scaling form for the osmotic pressure, diffusion measurements for higher-molecular-weight polymers, comparison of SANS radius of gyration results, and the validity of using diffusion measurements to estimate the overlap concentration.

[27] S. R. Kline, J. Appl. Crystallogr. 39, 895 (2006).

[28] P. N. Lavrenko, O. I. Mikriukova, and O. V. Okatova, Anal. Biochem. 166, 287 (1987).

[29] M. Daoud, J. P. Cotton, B. Farnoux, G. Jannink, G. Sarma, H. Benoit, C. Duplessix, C. Picot, and P. G. de Gennes, Macromolecules 8, 804 (1975).
[30] U. Zettl, S. T. Hoffmann, F. Koberling, G. Krausch, J. Enderlein, L. Harnau, and M. Ballauff, Macromolecules 42, 9537 (2009).

[31] S. Barhoum, S. Palit, and A. Yethiraj, Prog. Nucl. Magn. Reson. Spectrosc. 94-95, 1 (2016).

[32] P. T. Callaghan and D. N. Pinder, Macromolecules 14, 1334 (1981).

[33] Y. Rosenfeld, Phys. Rev. A 15, 2545 (1977).

[34] M. Dzugutov, Nature (London) 381, 137 (1996).

[35] A. L. Thorneywork, R. E. Rozas, R. P. A. Dullens, and J. Horbach, Phys. Rev. Lett. 115, 268301 (2015).

[36] G. D. Wignall, K. C. Littrell, W. T. Heller, Y. B. Melnichenko, K. M. Bailey, G. W. Lynn, D. A. Myles, V. S. Urban, M. V. Buchanan, D. L. Selby, and P. D. Butler, J. Appl. Crystallogr. 45, 990 (2012).

[37] W. Jiang, J. Huang, Y. Wang, and M. Laradji, J. Chem. Phys. 126, 044901 (2007).

[38] Y. Oono, J. Chem. Phys. 78, 520 (1983).

[39] C. Le Coeur, Ph.D. thesis, L'Université Pierre et Marie Curie, 2010. 